\title{
Facial root entry/exit zone contact in microvascular decompression for hemifacial spasm: a historical control study
}

\author{
Xianxia Yan^, Chengwen Ma, Junxiang Gu, Jianqiang Qu, Junjie Quan, Xi Zhang, Qin Song, Le Zhou^ \\ Department of Neurosurgery, the Second Affiliated Hospital of Xi'an Jiaotong University, Xi'an, China \\ Contributions: (I) Conception and design: X Yan, J Qu; (II) Administrative support: L Zhou; (III) Provision of study materials or patients: J Quan, X \\ Zhang, Q Song; (IV) Collection and assembly of data: C Ma, J Gu; (V) Data analysis and interpretation: X Yan; (VI) Manuscript writing: All authors; \\ (VII) Final approval of manuscript: All authors. \\ Correspondence to: Le Zhou. Department of Neurosurgery, the Second Affiliated Hospital of Xi'an Jiaotong University, No. 157 West Fifth Road, \\ Xi'an 710004, China. Email: drzhoule@163.com.
}

Background: Microvascular decompression (MVD) surgery is recognized as an effective treatment for hemifacial spasm (HFS). In MVD surgery, biocompatible materials are usually implanted in situ at the neurovascular conflict site in contact with the offending vessel and the facial root entry/exit zone (REZ). Another procedure of implanting the materials between the responsible vessel and the supraolivary fossa without REZ contact has also been applied. However, it is unclear whether there are any differences between these 2 procedures (REZ-contact procedure vs. REZ-non-contact procedure). Therefore, the aim of the present study was to investigate the effect of the placement of implants (contacting or not contacting the facial REZ) on surgical operations and outcomes

Methods: A historical control study was performed. Clinical data of HFS patients who underwent MVD between December 2016 and November 2018 were reviewed and categorized into 1 group with the REZcontact procedure or another group with the REZ-non-contact procedure according to the decompression strategy they received. Clinical demographics, postoperative outcomes, and complications were collected and compared between the two groups.

Results: Not all patients are suitable for REZ-non-contact decompression. A total of 205 patients were enrolled: 112 in the REZ-contact group and 93 in the REZ-non-contact group. In the early postoperative period, the complete cure rate in the REZ-non-contact group was significantly higher than that in the REZ-contact group. The reappearance and partial relief rates in the REZ-contact group were significantly higher than those in the REZ-non-contact group. The incidence of short-term neurological complications, especially hearing loss and transient facial palsy, was lower in the REZ-non-contact group ( $\mathrm{P}=0.043)$. But for long-term follow-up of $>1$ year, there was no significant difference between the two groups in either curative effects or neurological complications. The operating time for REZ-non-contact decompression was relatively longer than for REZ-contact decompression $(\mathrm{P}=0.000)$. An unexpected subdural hemorrhage occurred in the REZ-non-contact group.

Conclusions: REZ-non-contact decompression procedure showed superiority only in short-term postoperative outcomes. Given its limitations and potential risks, the REZ-non-contact procedure can be used as an alternative individualized strategy in MVD, and there is no need to pursue REZ-non-contact during the decompression.

Keywords: Hemifacial spasm (HFS); microvascular decompression (MVD); facial root entry/exit zone; implants; decompression procedure

Submitted Dec 14, 2020. Accepted for publication Mar 21, 2021.

doi: 10.21037/atm-20-7985

View this article at: http://dx.doi.org/10.21037/atm-20-7985

^ ORCID: Xianxia Yan, 0000-0002-9842-0438; Le Zhou, 0000-0001-6985-1979. 


\section{Introduction}

Primary hemifacial spasm (HFS) has been considered as a hyperactive facial nerve dysfunction triggered by offending vessels (1). Usually, the offending vessels compress the proximal area of facial nerve roots, an area known as the facial root entry/exit zone (REZ) which is fragile and prone to irritation (2-5). Wherefore, the microvascular decompression (MVD) surgery separating the offending vessel and facial REZ commonly makes an immediate recovery. In China, implant materials, such as polytetrafluoroethylene (Teflon) and polyester repair patches, are used in MVD surgery (6). Usually, the inserted materials are placed in situ at the neurovascular conflict site in contact with the facial REZ (6-9). However, numerous studies have reported that implantrelated adhesion, granuloma or malposition could be the cause for the persistence and recurrence of the spasm (10-21). To improve the decompression results, a procedure of placing the implants beyond the REZ by pushing offending vessels away from the facial nerve has been proposed (22). Two procedures currently exist for placement of implants, with the implant being in contact with the REZ in one procedure and not in contact in the other. However, there is a lack of published studies, especially large-sample cohort studies, that have comparatively analyzed these 2 procedures (REZ-contact vs. REZ-non-contact). At our institution, both procedures are used. Therefore, in the present study, we tend to provide a comprehensive analysis of these 2 procedures in terms of surgical operations and outcomes. We present the following article in accordance with the STROBE reporting checklist (available at http://dx.doi.org/10.21037/atm-20-7985).

\section{Methods}

\section{Study design}

The present study was a single-center, retrospective, historical control study conducted at the Department of Neurosurgery of the Second Affiliated Hospital of Xi'an Jiaotong University. The study was conducted in accordance with the Declaration of Helsinki (as revised in 2013). The study was approved by the hospital ethics committee (No. 2018-2123), and individual consent for this retrospective analysis was waived.

\section{Patient inclusion}

HFS patients who had received MVD surgery at our neurosurgical center between December 2016 and
November 2018 were included in the present study. During this period, a fixed medical team composed of the same neurosurgeons oversaw MVD surgeries. From the end of 2017 to the beginning of 2018, the REZ-non-contact procedure was prescribed for the decompression. Prior to that, the REZ-contact procedure had been used. The enrollment criteria were determined by which procedure the patients received. Patients who underwent MVD surgery prior to 2018 were most likely enrolled in the REZ-contact group, and those who underwent MVD surgery after this time were included in the REZ-non-contact group. To ensure the comparability of the neurovascular complexity between the two groups, cases in which neurovascular conflicts were beyond REZ and cases in which only the REZ-contact decompression could be performed due to the immovable structure of offending vessels were excluded. The exclusion criteria were as follows: (I) secondary facial spasm; (II) accompanied by other nervous system diseases; (III) neurovascular conflict site beyond REZ; (IV) immovable offending vessels; (V) patient failure to comply with a minimum follow-up of 2 years.

\section{Follow-up management}

The minimum follow-up period was set at 2 years, as most neurosurgeons believed that the final outcomes of MVD can be judged credibly after 1 year (23). All HFS patients in our center were advised of further checkups at regular intervals of $1,3,6$, and 12 months postoperatively. Most discharged patients agreed to the follow-up evaluations, including residual spasms, and hearing and facial movement tests. Telephone or WeChat interviews were used as alternatives for patients unable to attend in person because of distance, and for patients who were satisfied with their recovery and did not require long-term face-to-face follow-ups. A data set for follow-up patients was designed and made available for surgeons, so that they could make real-time updates during the follow-up visits.

\section{Surgical procedures}

All patients were treated by the same medical team, and all surgeries were performed by the same senior neurosurgeon at the same institution. Under general anesthesia, patients were positioned in the lateral park bench position, with the heads flexed and fixed. The suboccipital-retrosigmoid approach was applied in the following manner (6): a linear oblique skin incision was made posterior to the mastoid, and the 

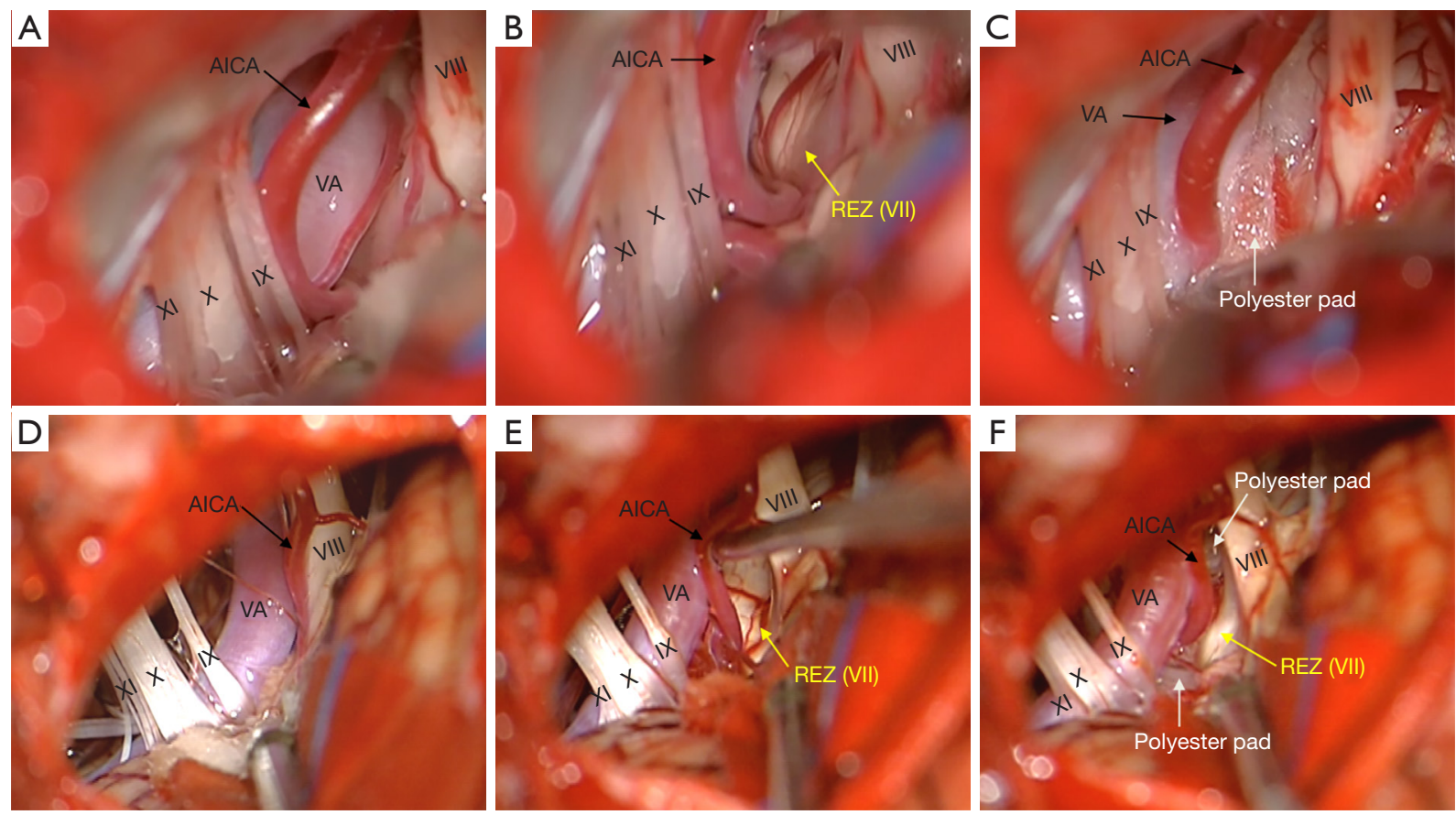

Figure 1 Intraoperative photos showing the 2 decompression procedures and implants placement. (A,B,C) REZ-contact procedure: (A) VA and AICA compress the facial REZ in a tandem pattern; (B) VA and AICA are nudged away from the REZ by a separator; (C) pieces of Polyester pads are inserted between the offending vessels and the REZ; (D,E,F) REZ-non-contact procedure: (D) VA and AICA tracking along the roots of facial-acoustic nerve complex (the VII cranial nerve is covered by the VIII); (E) VA and AICA are nudged away from the REZ by a separator; (F) pieces of properly sized polyester pads are inserted into the interspace between offending vessels and brainstem without contacting REZ. VA, vertebral artery; AICA, anterior inferior cerebellar artery; REZ, facial root entry/exit zone.

craniotomy via a small bone flap $(2 \mathrm{~cm} \times 2 \mathrm{~cm})$ was performed after musculoaponeurotic dissection and followed by a curved dural incision followed. After dissection the arachnoid membrane, retraction the flocculus, and gentle drainage of the cerebrospinal fluid, the facial REZ was exposed. Once the neurovascular conflict was confirmed, several polyester pads (CHEST, MedTech Co., Shanghai, China) were inserted between the facial nerve and the offending vessels with the REZ-contact decompression strategy (Figure 1A,B,C). In the REZ-non-contact procedure, any vessels relevant to the facial REZ were moved gently away from the REZ and fixed with well-sized polyester pads without contacting with REZ (Figure 1D,E,F). As for the cisternal segment of facial nerve beyond REZ, contact with the polyester pads was acceptable. In some cases, thick arachnoid trabeculae might tether the vessel tightly to the facial nerve, an arachnoid trabeculae debonding was needed before decompression. The REZ-non-contact decompression was inapplicable in cases where the offending vessel could not be moved because it went through the facial-vestibulocochlear nerve complex or had a tension perforator (24). According to the exclusion criteria, these cases $(n=9)$ were not included in the study. After adequate decompression and rechecking of the placement of the polyester pads, watertight dural closure was performed using artificial dura, and mastoid cells were sealed if necessary. Finally, the periosteum, muscle and cutaneous flaps were carefully sutured in layers. The same procedures for craniotomy and closure were adopted in these 2 procedures. During the surgeries, the brainstem auditory evoked potentials, lateral spread response (LSR), and facial motor evoked potentials were monitored using an intraoperative electrophysiological monitor (Cadwell Laboratories, Kennewick, WA, USA), and the disappearance of LSR was used as a crucial indication for decompression completion.

\section{Data collection}

Two datasets were designed for data collection. One was used to extract data from the medical records of the enrolled 
patients, including demographics, surgical procedures, surgical videos, immediate postoperative outcomes, and complications. The other was used for collecting and updating follow-up information which contains the checkups of re-visiting patients, telephone or WeChat interview records, and other online consultations. Data collection was performed by 2 researchers and was crosschecked by another.

\section{Statistical analysis}

Statistical analyses were performed with SPSS version 23.0 (IBM Corp., Armonk, NY, USA). Continuous variables are expressed as mean \pm standard deviation or median/ (minimum-maximum) in accordance with their distribution, and were compared using independent $t$-test or MannWhitney $U$ test accordingly. Categorical variables are presented as numbers (\%) and were evaluated using $\chi^{2}$-test, in cases where expected counts in any cells were $<5$, Fisher's exact test was used instead. All reported $\mathrm{P}$ values were two-sided, and a $\mathrm{P}$ value $<0.05$ was considered statistically significant.

\section{Results}

\section{Patient demographics}

A total of 229 patients who underwent MVD for HFS were initially included. Of these, 24 patients were excluded because they also had cerebrovascular malformation $(n=1)$, Chiari malformation $(\mathrm{n}=1)$, or trigeminal neuralgia $(\mathrm{n}=1)$; and were only available for REZ-contacted decompression $(\mathrm{n}=9)$; had a neurovascular conflict site located beyond the REZ ( $n=7)$; or were lost to follow-up (n=5). Finally, 205 patients were enrolled in the present study: 112 in the REZ-contact group and 93 in the REZ-non-contact group (Figure 2).

Patient characteristics are presented in Table 1. Clinical features, including sex, age, affected side, duration of symptoms, composition of offending vessels, and LSR disappearance, were not statistically different between the two groups. However, the total operation time was significantly longer in the REZ-non-contact group $(\mathrm{P}=0.000)$.

\section{Clinical outcomes}

As is shown in Figures 3,4, the immediate postoperative outcomes were categorized as complete cure, partial relief, and ineffectiveness, according to spasm remission. One week postoperatively, a new subcategory of reappearance was added. This category comprised patients who experienced spasm reappearance after the initial complete relief.

The postoperative complete cure rates immediately after surgery, and 1 week, $1,3,6$, and 12 months postoperatively were 91 (81.3\%), 81 (72.3\%), 92 (82.1\%), 95 (84.8\%), 98 (87.5\%), and $101(90.2 \%)$, respectively, in the REZ-contact group, and 86 (92.5\%), 82 (88.2\%), 86 (92.5\%), 87 (93.5\%), 87 (93.5\%), and 87 (93.5\%), respectively, in the REZnon-contact group (Table S1). With the exception for an outbreak of reappearance within 1 week after surgery, the symptom of residual spasm in both groups continued to be relieved within 1 year. The complete cure rates immediately after surgery, and 1 week, 1 month, and 3 months in the REZ-non-contact group were significantly higher than that in the REZ-contact group $(\mathrm{P}=0.020, \mathrm{P}=0.005, \mathrm{P}=0.029$, $\mathrm{P}=0.049$, respectively). But 3 months later, there was no significant difference in complete cure rate between the two groups (Table S2 and Figure 5).

Partial relief, sometimes recognized as delayed cure, was relatively higher immediately after surgery $[\mathrm{n}=19(17.0 \%)$ in the REZ-contact group and $\mathrm{n}=6(6.5 \%)$ in the REZ-noncontact group; Table S3]. With the prolongation of 2-year follow-up, 16 of 20 patients $(80.0 \%)$ in the REZ-contact group and 6 of 7 patients (85.7\%) in the REZ-non-contact group were completely cured (Figures 3,4). Although the partial relief rate in the REZ-contact group was significantly higher immediately after surgery $(\mathrm{P}=0.022)$, during the subsequent follow-up period, there was no significant difference between the two groups (Table S3 and Figure 6).

Among the patients who recovered immediately after surgery, 17 (18.7\%) in the REZ-contact group and 7 (8.1\%) in the REZ-non-contact group experienced reappearance within 1 week (Figures 3,4; Table S4). The cumulative reappearance rate in the REZ-contact group was significantly higher at 1 week, 1 month, and 12 months after the surgery (Table S4 and Figure 7). However, during the subsequent 1-year follow-up, 16 (94.1\%) patients in the REZ-contact group and $6(85.7 \%)$ patients in the REZ-non-contact group achieved complete recovery again (Figures 3,4). The remaining 1 patient in the REZ-contact group and 1 patient in the REZ-non-contact group suffered a sustained but alleviated spasm, these 2 cases should be interpreted as partial relief. Another 4 patients in the REZ-contact group and 3 patients in the REZ-non-contact group underwent delayed recurrence 1 month after surgery and had no signs of recovery. In each group, 2 patients relapsed after 1 year 


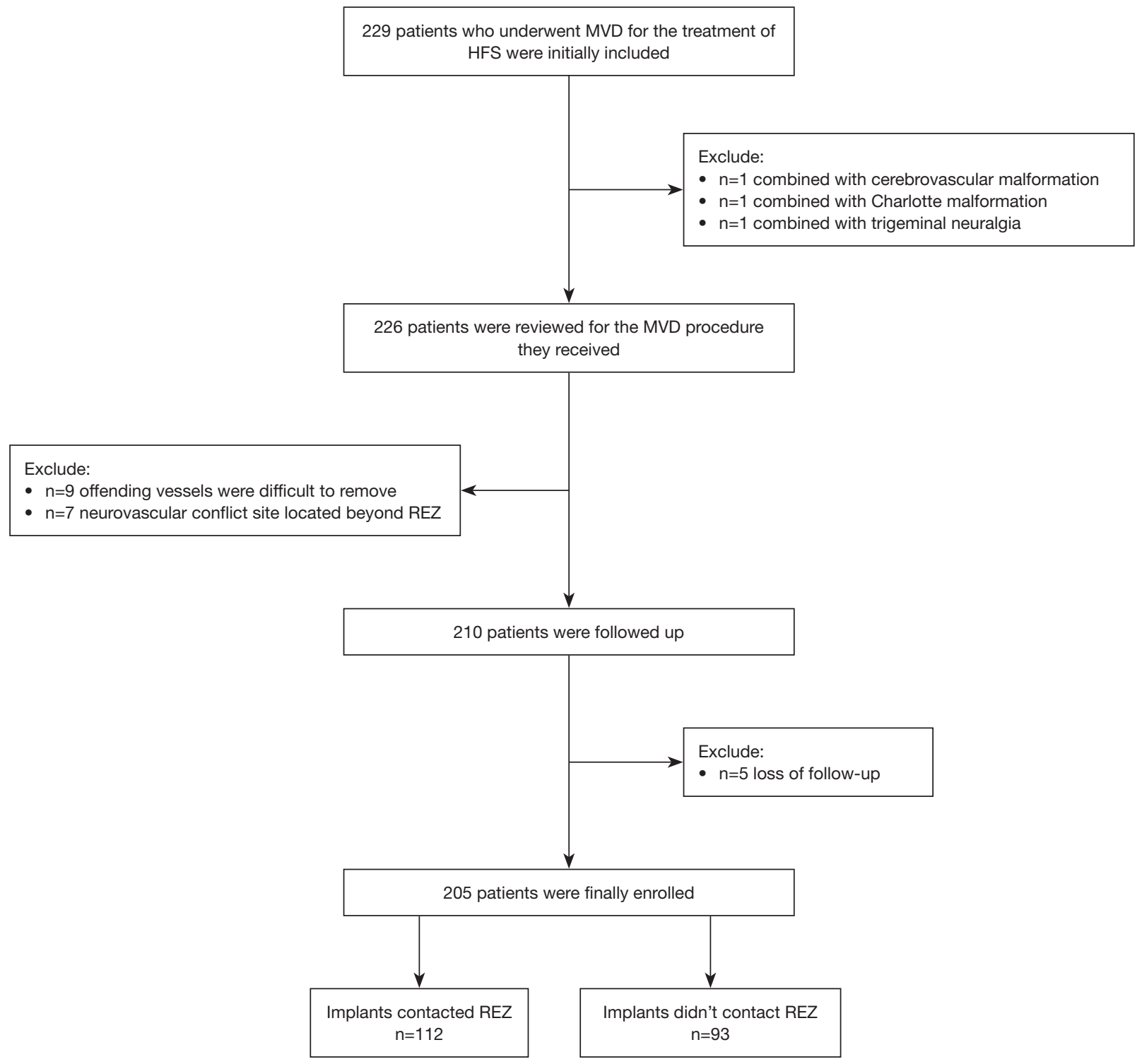

Figure 2 Schema of patient recruitment process. MVD, microvascular decompression; HFS, hemifacial spasm; REZ, facial root entry/exit zone.

(Figures 3,4).

\section{Discomforts and complications}

Besides wound pain, fever and vomiting are the most common discomforts after MVD. In the REZ-contact group and REZ-non-contact group, the incidence of fever was $41.1 \%$ and $32.3 \%$, respectively, and the incidence of vomiting was $19.6 \%$ and $24.7 \%$, respectively. The incidence of fever and vomiting between the two groups was not significantly different (Table 2).

Hearing loss reported in this study may be mostly conductive, as the patients complained of muffled or garbled sound and the postoperative computerized tomography showed liquid density in mastoid air cells. The incidence of early hearing loss in the REZ-contact group (5.4\%) was relatively higher than that in the REZ-non-contact group (1.1\%). Except for two patients who developed persistent hearing loss in the REZ-contact group, the other patients gradually recovered within one year (Table 2).

Most facial palsy in this study occurred within one week after surgery. And patients with mild facial palsy (HouseBrackmann grade II) only showed flattened nasolabial fold. More patients in the REZ-contact group (10.7\% vs. $4.3 \%)$ 
Table 1 Clinical features

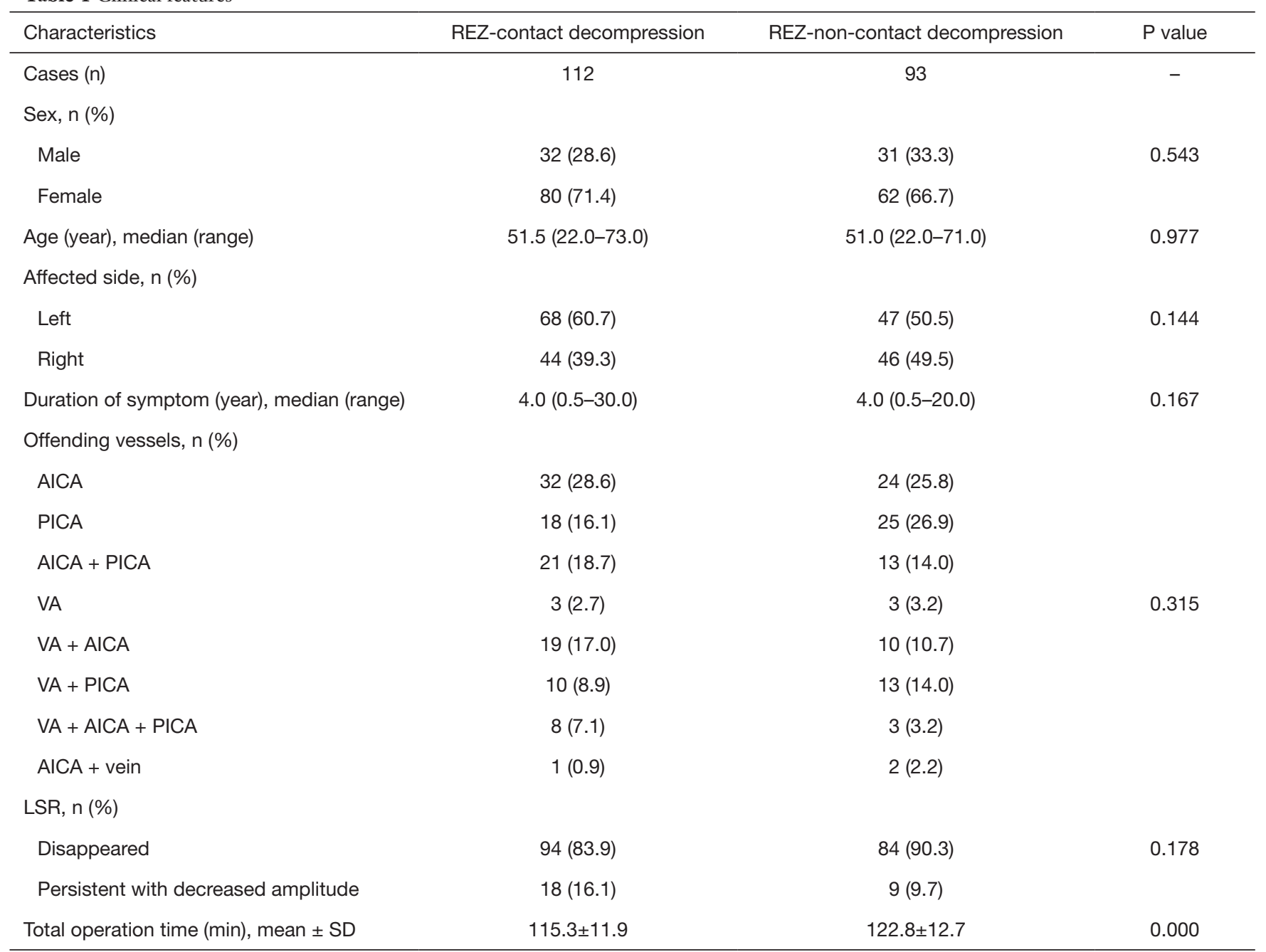

AICA, anterior inferior cerebellar artery; PICA, posterior inferior cerebellar artery; VA, vertebral artery; LSR, lateral spread response; SD, standard deviation; REZ, facial root entry/exit zone.

encountered mild facial palsy. Two cases with severe facial palsy (House-Brackmann grade IV) occurred 14 days (REZcontact group) and 17days (REZ-non-contact group) after surgery respectively. All facial palsy, no matter mild or severe, disappeared within one year after surgery. One patient (1.1\%) in the REZ-non-contact group encountered a subdural hemorrhage and received hematoma removal surgery (Table 2).

\section{Discussion}

\section{Clinical outcomes}

Facial REZ is commonly defined as a proximal segment from the facial root exit point to the transition zone
$(2,25,26)$. With oligodendrocyte-derived myelin, the REZ is structurally weaker and more vulnerable to the influence of vascular compression $(5,27)$. Complete relief of HFS is dependent on sufficient decompression during MVD (28-30). Theoretically, as a radical and complete decompression approach that fully isolates offending vessels from facial REZ, the REZ-non-contact procedure should be more effective in decompression. One published study has reported that placing the implants between offending vessels and the brainstem without REZ contact obtained satisfactory results (22). The findings of the present study also supported that the REZ-non-contact procedure has superiority in short-term outcomes.

Not all cases are suitable for the REZ-non-contact 


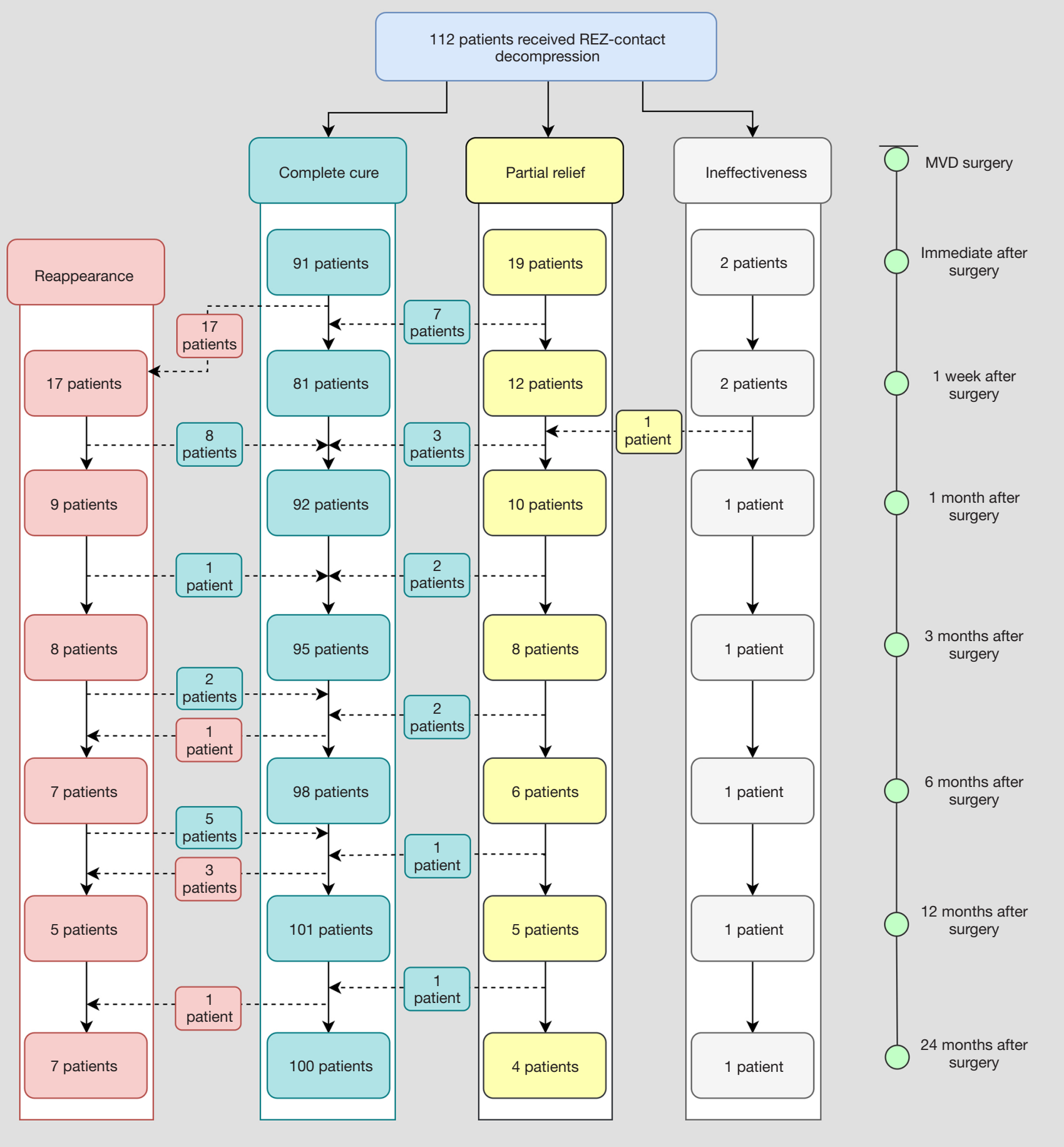

Figure 3 Diagram showing the categorization and progression of clinical outcomes after REZ-contact decompression. MVD, microvascular decompression; REZ, facial root entry/exit zone. 


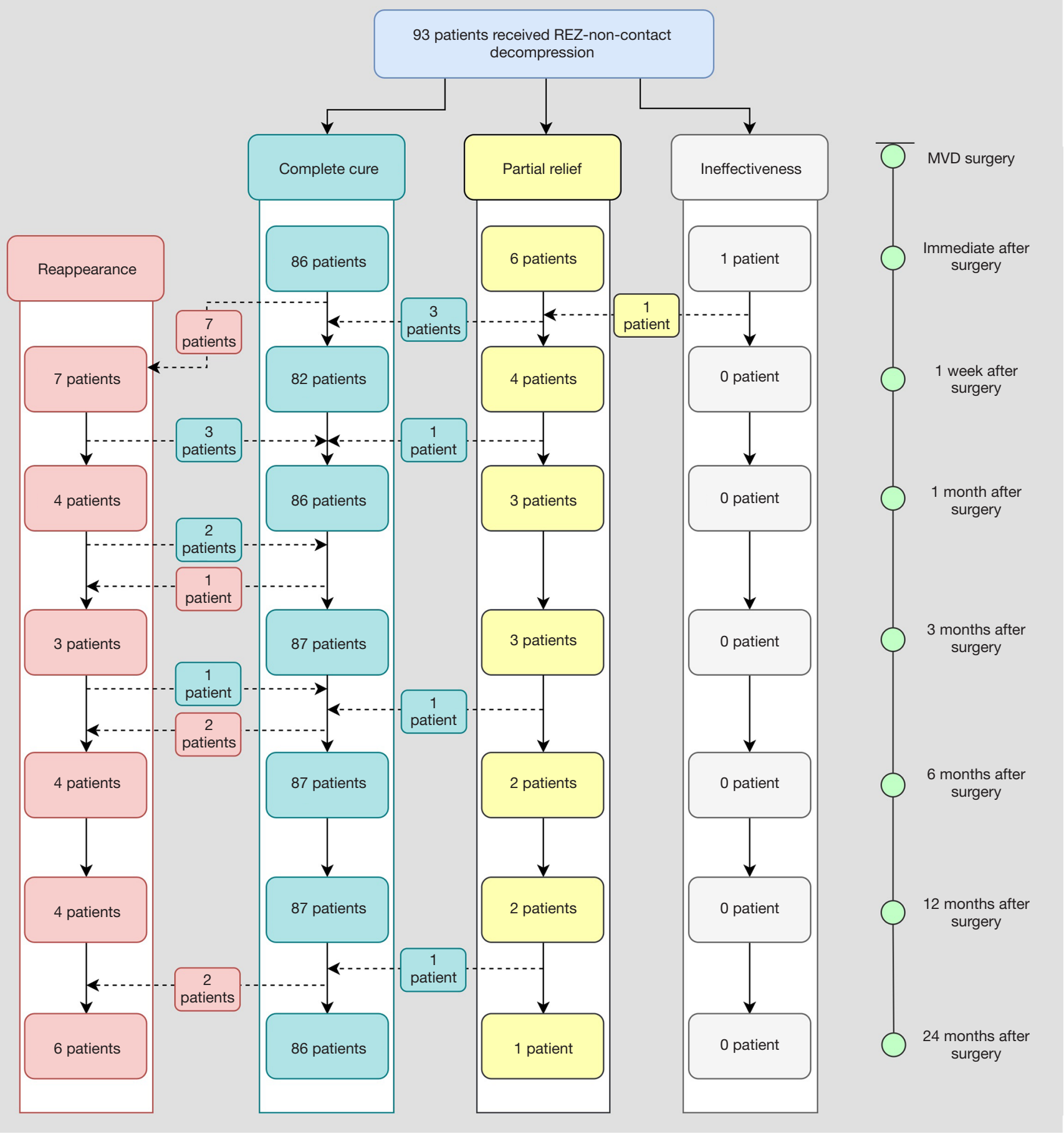

Figure 4 Diagram showing the categorization and progression of clinical outcomes after REZ-non-contact decompression. MVD, microvascular decompression; REZ, facial root entry/exit zone. 


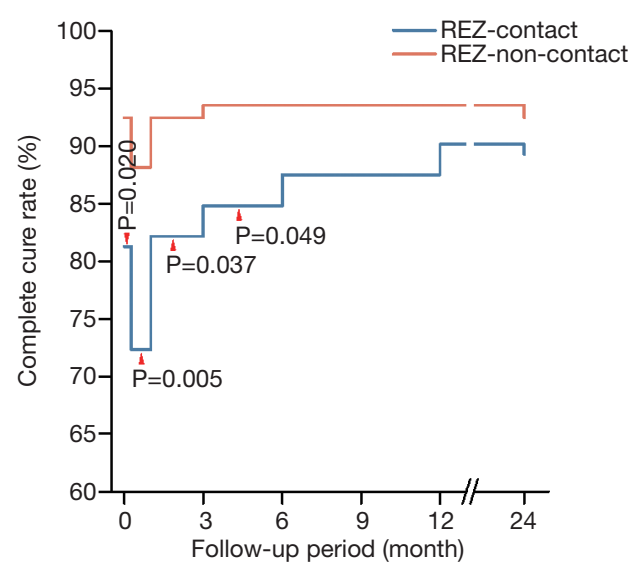

Figure 5 Line chart showing complete cure rate in the two groups. The brown triangles represent significant differences. REZ, facial root entry/exit zone.

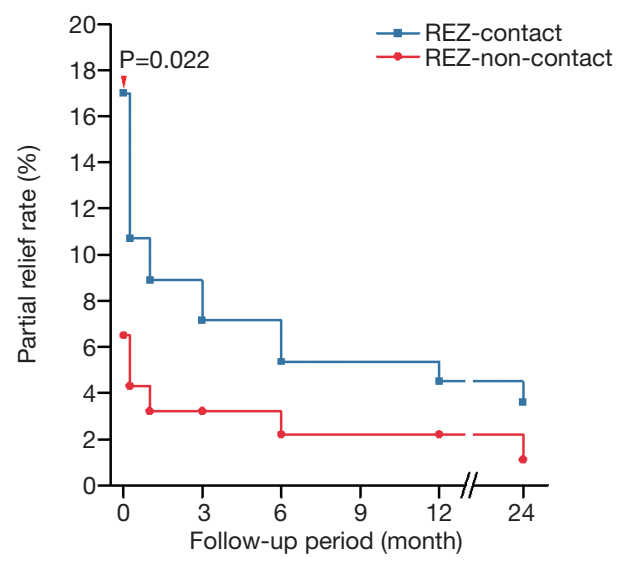

Figure 6 Line chart showing partial relief rate in the two groups. The red triangle represents significant difference. REZ, facial root entry/exit zone.

procedure. In some situations where the perforating vessels are attached too firmly to, or even penetrate into, the facial-acoustic nerve, the REZ-non-contacted procedure is inapplicable. In the present study, there were 9 cases in which the offending vessels could not be removed, and a thorough decompression was unattainable. The complex structure of responsible vessels might have affected the MVD outcomes, regardless of which compression procedure was used, so these cases were excluded from the present study. In comparing the length of the time spent on these 2 procedures, we did not record the operation time under the microscope although it is more appropriate to do so. As craniotomy and suture were performed by the same

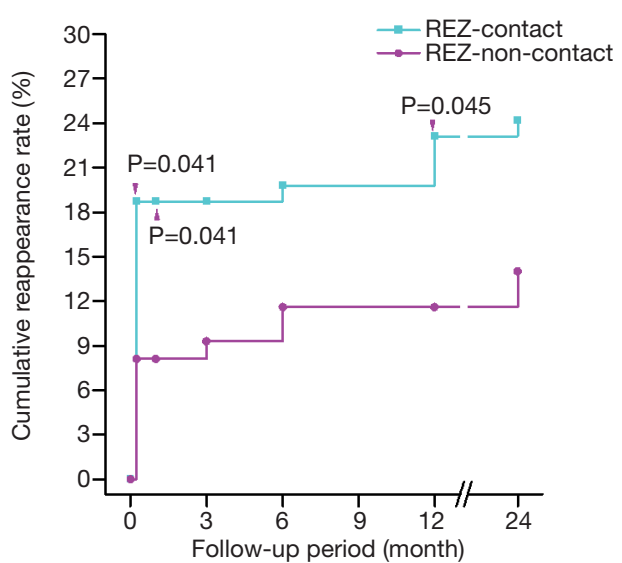

Figure 7 Line chart showing cumulative reappearance rate in the two groups. The magenta triangles represent significant differences. REZ, facial root entry/exit zone.

assistants, which eliminated the variations in operating time caused by inconsistent surgical skills, the entire operation time could be used, to some extent, to represent the exact time spent on these 2 procedures. For the REZ-non-contact procedure, it takes more time to remove the offending vessel and adjust implanted pads to fix the offending vessel.

Each group experienced a small outbreak of reappearance 1 week after surgery, most likely due to the irritation of implants or early recontact of the offending vessels. The incidence of reappearance was relatively lower in the REZ-non-contact group, which indicates that avoiding implants contact with REZ may be effective in reducing this early reappearance. Of course, the increasing sophistication of surgical skills may might have also contributed to this reduction.

As most of the patients who suffered early spasm reappearance achieved complete cure again within 1 year, we used the term reappearance instead of recurrence to describe this phenomenon. In the reappearance category, 4 patients in the REZ-contact group and 3 patients in the REZ-noncontact group had delayed recurrence 1 month after surgery, but had no signs of recovery 2 years postoperatively. These 7 cases were considered to be cases of recurrence. With the other 2 cases of recurrence 1 year after surgery in each group, there were 11 patients with recurrence: 6 in the REZ-contact group and 5 in the REZ-non-contact group, thus, there was no significant difference between the two groups in recurrence.

\section{Discomforts and complications}

Fever and vomiting mainly occur in the first 1 or 2 days 
Table 2 Postoperative discomforts and complications

\begin{tabular}{|c|c|c|c|}
\hline Discomforts/complications & REZ-contact decompression, n (\%) & REZ-non-contact decompression, n (\%) & $P$ value \\
\hline \multicolumn{4}{|l|}{ Fever } \\
\hline No & $66(58.9)$ & $63(67.7)$ & \multirow[t]{2}{*}{0.193} \\
\hline Yes (axillary temperature $>37.2^{\circ} \mathrm{C}$ ) & $46(41.1)$ & $30(32.3)$ & \\
\hline \multicolumn{4}{|l|}{ Vomiting } \\
\hline No & $90(80.4)$ & $70(75.3)$ & \multirow[t]{2}{*}{0.381} \\
\hline Yes & $22(19.6)$ & $23(24.7)$ & \\
\hline \multicolumn{4}{|c|}{ Neurological complications (1 month after surgery) } \\
\hline No & $93(83.0)$ & $86(92.5)$ & \\
\hline Mild facial palsy & $12(10.7)$ & $4(4.3)$ & \multirow{3}{*}{0.043} \\
\hline Severe facial palsy & $1(0.9)$ & $1(1.1)$ & \\
\hline Subdural hematoma & $0(0.0)$ & $1(1.1)$ & \\
\hline \multicolumn{4}{|c|}{ Neurological complications (1 year after surgery) } \\
\hline No & $110(98.2)$ & $93(100.0)$ & \\
\hline \multicolumn{4}{|l|}{ Yes } \\
\hline Hearing loss & $2(1.8)$ & $0(0.0)$ & \multirow[t]{2}{*}{0.502} \\
\hline Mild facial palsy & $0(0.0)$ & $0(0.0)$ & \\
\hline
\end{tabular}

REZ, facial root entry/exit zone.

postoperatively, and are mostly due to surgical trauma, implant material stimulation, and general anesthesia.

Hearing loss, as well as facial palsy is commonly reported after MVD surgery (31-36). The type of hearing loss in the present study could not be well defined, as the pure tone audiometry was not performed in every patient. However, the postoperative computerized tomography showed destruction and hydrops in mastoid air cells in patients with hearing loss. It has been reported that fluid entering the mastoid air cells and or bone-dust deposition during a craniotomy may result in conductive hearing loss (31,37-39). Avoiding open or effective closure of the mastoid antrum during craniotomy could prevent conducted hearing loss postoperatively (40-42). In the last 2 or 3 years, we did take measures to prevent fluid from entering the mastoid air cells. So, the lower incidence of hearing loss in the REZ- non-contact group may be due to the modified craniotomy. As to sensorineural hearing loss, it may result from stretching of the vestibulocochlear nerve or direct trauma to the nerve (43). Therefore, hearing loss may be more related to the surgical manipulation, but not to the placement of the implants.

Facial weakness that occurs $>24$ hours after MVD can be defined as delayed facial palsy. However, the reported onset time, duration, as well as the severity of delayed facial palsy, varied in different studies. Liu et al. reported the onset time ranged from 0 to 30 days, and the duration from 10 to 230 days (44). Hua et al. reported the onset time ranged from 3 to 16 days, and the duration from 15 to 136 days; $29.41 \%$ patients had grade II palsy, $44.12 \%$ patients had grade III, $14.71 \%$ patients had grade IV, $11.76 \%$ patients had grade $\mathrm{V}$ according to the House-Brachmann classification (45). 
Rhee et al. reported the onset of palsy between 7 and 23 days and a recovery time from 25 days to 17 weeks; $61 \%$ patients had grade II palsy, 29\% patients had grade III and $10 \%$ patients had grade IV (34). The onset time of facial palsy in the present study was mostly within 1 week after surgery, earlier than the reported. Twelve of 13 (92.3\%) patients in the REZ-contact group and 4 of $5(80.0 \%)$ patients in the REZ-non-contact group had grade II palsy, the degree of palsy was milder than the reported. Many HFS patients in China receive acupuncture treatment before MVD surgery. Acupuncture would not cause symptoms of palsy, but the muscle weakness and a decreased amplitude of facial compound motor action potential was reported (46). Thus, the existed facial weakness may be related to the early onset and the mildness of facial palsy. Heterogeneous stimulation by implants can directly irritate the facial nerve roots and induce facial palsy (47). Therefore, the lower incidence of facial palsy in the REZ-non-contact group may be due to the REZ-non-contact procedures. In addition to the reasons presumed above, other possible mechanisms causing facial palsy, such as viral reactivation (48-50), and microcirculation disturbance due to vasospasm $(33,51)$, are also concerned. But all these proposed mechanisms remain unproven.

Unlike other complications, subdural hemorrhage or hematoma is rare but fatal (52). Manipulations during surgery, such as brain retraction and unexplained rupture of the temporoparietal vessels, may be partly responsible for subdural hemorrhage or hematoma, especially in elderly patients (53). In the REZ-non-contact cohort, 1 patient developed a subdural hematoma 48 hours after MVD surgery. It is undeniable that the offending vessels in this procedure are usually moved away from the facial nerve and the implants sometimes need to be adjusted more often, which could, to some extent, increased the disturbance of offending vessels, and increasing the risk of intracranial hemorrhage. Of course, hypertension accompanied by atherosclerosis could also increase this risk (54).

The texture of polyester patch we used for MVD in the present study was a bit rough. Although its safety and effectiveness as an implant have been fully verified, it was possible that, in the REZ-contact group, the harder texture of polyester patch could have irritated the facial REZ and affected the short-term outcomes. But in fact, compared with studies using Teflon as the implants, the complete cure rate in the REZ-contact group in the present study was similar to or even higher $(36,55)$. The incidence of transient hearing loss, facial palsy in the REZ-contact group was close to or lower than the incidence reported in other large- sample studies $(33,36,56)$. That is, the polyester patch is not inferior to Teflon in terms of effectiveness and safety.

Finally, the inherent bias in this work is inevitable because of its retrospective study design and the increasing surgical proficiency. Therefore, further prospective investigations on larger samples with long-term follow-ups are recommended to assess long-term outcomes of these procedures.

\section{Conclusions}

The REZ-non-contact procedure demonstrates superiority in short-term postoperative outcomes by improving the immediate cure rate and reducing the short-term neurological complications. However, long-term outcomes, including complete cure rate, recurrence, and neurological complications, were similar between the REZ-non-contact procedure and the REZ-contact procedure. Because of the complexity of the neurovascular structure, not all patients are suitable for REZ-non-contact decompression. The transposition of offending vessels during this procedure may cause potential risk of hemorrhage. Given the limitations and potential risks, the REZ-non-contact procedure can be used as an alternative individualized strategy but is unsuitable to act as a guide principle for implant placement.

\section{Acknowledgments}

We especially want to thank Wei Wu for statistical supports, and R. Scott, J. Gray, and Jin Li for language help.

Funding: This study was supported by the National Natural Science Foundation of China (No. 81801121) and the Key Research and Development Programs of Shaanxi Provence, China (No. 2017SF-026 and 2016KTCL03-08).

\section{Footnote}

Reporting Checklist: The authors have completed the STROBE reporting checklist. Available at http://dx.doi. org/10.21037/atm-20-7985

Data Sharing Statement: Available at http://dx.doi. org/10.21037/atm-20-7985

Peer Review File: Available at http://dx.doi.org/10.21037/ atm-20-7985

Conflicts of Interest: All authors have completed the ICMJE 
uniform disclosure form (available at http://dx.doi. org/10.21037/atm-20-7985). The authors have no conflicts of interest to declare.

Ethical Statement: The authors are accountable for all aspects of the work in ensuring that questions related to the accuracy or integrity of any part of the work are appropriately investigated and resolved. The study was conducted in accordance with the Declaration of Helsinki (as revised in 2013). The study was approved by the hospital ethics committee (no. 2018-2123), and individual consent for this retrospective analysis was waived.

Open Access Statement: This is an Open Access article distributed in accordance with the Creative Commons Attribution-NonCommercial-NoDerivs 4.0 International License (CC BY-NC-ND 4.0), which permits the noncommercial replication and distribution of the article with the strict proviso that no changes or edits are made and the original work is properly cited (including links to both the formal publication through the relevant DOI and the license). See: https://creativecommons.org/licenses/by-nc-nd/4.0/.

\section{References}

1. Lefaucheur JP. New insights into the pathophysiology of primary hemifacial spasm. Neurochirurgie 2018;64:87-93.

2. Campos-Benitez M, Kaufmann AM. Neurovascular compression findings in hemifacial spasm. J Neurosurg 2008;109:416-20.

3. Zhong J, Zhu J, Sun H, et al. Microvascular decompression surgery: surgical principles and technical nuances based on 4000 cases. Neurol Res 2014;36:882-93.

4. Zhong J, Li ST, Zhu J, et al. Is entire nerve root decompression necessary for hemifacial spasm? Int J Surg 2011;9:254-7.

5. De Ridder D, Moller A, Verlooy J, et al. Is the root entry/exit zone important in microvascular compression syndromes? Neurosurgery 2002;51:427-33; discussion 433-4.

6. Sindou M, Mercier P. Microvascular decompression for hemifacial spasm: Surgical techniques and intraoperative monitoring. Neurochirurgie 2018;64:133-43.

7. Qi H, Zhang W, Zhang X, et al. Microvascular Decompression Surgery for Hemifacial Spasm. J Craniofac Surg 2016;27:124-7.

8. Ghali MGZ, Srinivasan VM, Viswanathan A. Microvascular Decompression for Hemifacial Spasm. Int
Ophthalmol Clin 2018;58:111-21.

9. Barker FG, 2nd, Jannetta PJ, Bissonette DJ, et al. Microvascular decompression for hemifacial spasm. J Neurosurg 1995;82:201-10.

10. Amador N, Pollock BE. Repeat posterior fossa exploration for patients with persistent or recurrent idiopathic trigeminal neuralgia. J Neurosurg 2008;108:916-20.

11. Capelle HH, Brandis A, Tschan CA, et al. Treatment of recurrent trigeminal neuralgia due to Teflon granuloma. J Headache Pain 2010;11:339-44.

12. Dou NN, Zhong J, Liu MX, et al. Teflon Might Be a Factor Accounting for a Failed Microvascular Decompression in Hemifacial Spasm: A Technical Note. Stereotact Funct Neurosurg 2016;94:154-8.

13. Chen J, Lee S, Lui T, et al. Teflon granuloma after microvascular decompression for trigeminal neuralgia. Surg Neurol 2000;53:281-7.

14. Oda K, Higuchi T, Murai Y, et al. Teflon granuloma after microvascular decompression for hemifacial spasm: a case report and literature review. Neurosurg Rev 2017;40:513-6.

15. Deep NL, Graffeo CS, Copeland WR 3rd, et al. Teflon granulomas mimicking cerebellopontine angle tumors following microvascular decompression. Laryngoscope 2017;127:715-9.

16. Rath SA, Klein HJ, Richter HP. Findings and long-term results of subsequent operations after failed microvascular decompression for trigeminal neuralgia. Neurosurgery 1996;39:933-8; discussion 938-40.

17. Kureshi SA, Wilkins RH. Posterior fossa reexploration for persistent or recurrent trigeminal neuralgia or hemifacial spasm: surgical findings and therapeutic implications. Neurosurgery 1998;43:1111-7.

18. Matsushima T, Yamaguchi $\mathrm{T}$, Inoue TK, et al. Recurrent trigeminal neuralgia after microvascular decompression using an interposing technique. Teflon felt adhesion and the sling retraction technique. Acta Neurochir (Wien) 2000;142:557-61.

19. Vitali AM, Sayer FT, Honey CR. Recurrent trigeminal neuralgia secondary to Teflon felt. Acta Neurochir (Wien) 2007;149:719-22; discussion 722.

20. Park YS, Chang JH, Cho J, et al. Reoperation for persistent or recurrent hemifacial spasm after microvascular decompression. Neurosurgery 2006;58:1162-7; discussion $1162-7$.

21. Ravina K, Strickland BA, Rennert RC, et al. Revision Microvascular Decompression for Trigeminal Neuralgia and Hemifacial Spasm: Factors Associated with Surgical 
Failure. J Neurol Surg B Skull Base 2019;80:31-9.

22. Nan-Xiang X, Lv-An C, Zhi-Jun C, et al. Placement of Teflon Sponges in Microvascular Decompression Procedure for Treatment of Hemifacial Spasm. J Neurol Surg A Cent Eur Neurosurg 2016;77:321-5.

23. Sindou M, Mercier P. Microvascular decompression for hemifacial spasm: Outcome on spasm and complications. A review. Neurochirurgie 2018;64:106-16.

24. Amagasaki K, Watanabe S, Naemura K, et al. Transposition of the Culprit Artery Passing Between the Facial Nerve and Auditory Nerve in Microvascular Decompression Surgery for Hemifacial Spasm. World Neurosurg 2019;127:e996-1002.

25. Guclu B, Sindou M, Meyronet D, et al. Cranial nerve vascular compression syndromes of the trigeminal, facial and vago-glossopharyngeal nerves: comparative anatomical study of the central myelin portion and transitional zone; correlations with incidences of corresponding hyperactive dysfunctional syndromes. Acta Neurochir (Wien) 2011;153:2365-75.

26. Eidelman BH, Nielsen VK, Moller M, et al. Vascular compression, hemifacial spasm, and multiple cranial neuropathy. Neurology 1985;35:712-6.

27. Yee GT, Yoo CJ, Han SR, et al. Microanatomy and histological features of central myelin in the root exit zone of facial nerve. J Korean Neurosurg Soc 2014;5 5:244-7.

28. Shin HS, Lee SH, Ko HC, et al. Evaluating Transient Hemifacial Spasm that Reappears After Microvascular Decompression Specifically Focusing on the Real Culprit Location of Vascular Compression. World Neurosurg 2017;98:774-9.

29. Jannetta PJ, Abbasy M, Maroon JC, et al. Etiology and definitive microsurgical treatment of hemifacial spasm. Operative techniques and results in 47 patients. J Neurosurg 1977;47:321-8.

30. Jannetta PJ. Trigeminal neuralgia and hemifacial spasm-etiology and definitive treatment. Trans Am Neurol Assoc 1975;100:89-91.

31. Ying T, Thirumala P, Gardner P, et al. The Incidence of Early Postoperative Conductive Hearing Loss after Microvascular Decompression of Hemifacial Spasm. J Neurol Surg B Skull Base 2015;76:411-5.

32. Lee MH, Jee TK, Lee JA, et al. Postoperative complications of microvascular decompression for hemifacial spasm: lessons from experience of 2040 cases. Neurosurg Rev 2016;39:151-8; discussion 158.

33. Lee JM, Park HR, Choi YD, et al. Delayed facial palsy after microvascular decompression for hemifacial spasm: friend or foe? J Neurosurg 2018;129:299-307.

34. Rhee DJ, Kong DS, Park K, et al. Frequency and prognosis of delayed facial palsy after microvascular decompression for hemifacial spasm. Acta Neurochir (Wien) 2006;148:839-43; discussion 843.

35. Zhao H, Zhang X, Tang YD, et al. Operative Complications of Microvascular Decompression for Hemifacial Spasm: Experience of 1548 Cases. World Neurosurg 2017;107:559-64.

36. Hyun SJ, Kong DS, Park K. Microvascular decompression for treating hemifacial spasm: lessons learned from a prospective study of 1,174 operations. Neurosurg Rev 2010;33:325-34; discussion 334.

37. Brooks RC, Graham MD. Postoperative accumulation of bone in the middle ear: effect on hearing and surgical implications. Am J Otol 1980;2:168-9.

38. Murai K, Kon Y, Obara Y, et al. A study on auditory disturbances after microvascular decompression for hemifacial spasm. Nihon Jibiinkoka Gakkai Kaiho 1991;94:657-66.

39. Devesa PM, Michaels L, Wright A. Ossicular fixation caused by bone dust after saccus decompression surgery. Otol Neurotol 2002;23:949-51; discussion 951-2.

40. Parikh AA, Brookes GB. Conductive hearing loss following retrolabyrinthine surgery. Arch Otolaryngol Head Neck Surg 1996;122:841-3.

41. Kim L, Wisely CE, Dodson EE. Transmastoid approach to spontaneous temporal bone cerebrospinal fluid leaks: hearing improvement and success of repair. Otolaryngol Head Neck Surg 2014;150:472-8.

42. Lin J, Zhang Y, Peng R, et al. Preoperative Imaging and Microscopic Navigation During Surgery Can Avoid Unnecessarily Opening the Mastoid Air Cells Through Craniotomy Using the Retrosigmoid Approach. World Neurosurg 2019;121:e15-21.

43. Thirumala P, Meigh K, Dasyam N, et al. The incidence of high-frequency hearing loss after microvascular decompression for trigeminal neuralgia, glossopharyngeal neuralgia, or geniculate neuralgia. J Neurosurg 2015;123:1500-6.

44. Liu LX, Ren YM, Ren PW, et al. Prognosis of Symptoms and Complications After Microvascular Decompression for Hemifacial Spasm: A Single-Center Experience. World Neurosurg 2018;118:e557-61.

45. Hua Z, Da TY, Hui WX, et al. Delayed Facial Palsy After Microvascular Decompression for Hemifacial Spasm. J Craniofac Surg 2016;27:781-3.

46. Zhang WB, Min LZ, Zhong WX, et al. Surgical effect 


\section{Page 14 of 14}

and electrophysiological study of patients with hemifacial spasm treated with botulinum toxin or acupuncture before microvascular decompression. Clin Neurol Neurosurg 2019;184:105417.

47. Kong CC, Guo ZL, Xu XL, et al. Delayed Facial Palsy After Microvascular Decompression for Hemifacial Spasm. World Neurosurg 2020;134:e12-5.

48. Gyo K, Honda N. Delayed facial palsy after middleear surgery due to reactivation of varicella-zoster virus. J Laryngol Otol 1999;113:914-5.

49. Furuta Y, Ohtani F, Fukuda S, et al. Reactivation of varicella-zoster virus in delayed facial palsy after dental treatment and oro-facial surgery. J Med Virol 2000;62:42-5.

50. Gianoli GJ. Viral titers and delayed facial palsy after acoustic neuroma surgery. Otolaryngol Head Neck Surg 2002;127:427-31.

51. Scheller C, Strauss C, Fahlbusch R, et al. Delayed facial nerve paresis following acoustic neuroma resection and

Cite this article as: Yan X, Ma C, Gu J, Qu J, Quan J, Zhang X, Song Q, Zhou L. Facial root entry/exit zone contact in microvascular decompression for hemifacial spasm: a historical control study. Ann Transl Med 2021;9(10):834. doi: 10.21037/atm-20-7985

\section{Yan et al. Two decompression strategies in MVD for HFS}

postoperative vasoactive treatment. Zentralbl Neurochir 2004;65:103-7.

52. Li N, Zhao W, Pu C, et al. Delayed hemorrhage following microvascular decompression. Three case reports. Neurol Med Chir (Tokyo) 2007;47:186-8.

53. Xia L, Liu MX, Zhong J, et al. Fatal complications following microvascular decompression: could it be avoided and salvaged? Neurosurg Rev 2017;40:389-96.

54. Seifman MA, Lewis PM, Rosenfeld JV, et al. Postoperative intracranial haemorrhage: a review. Neurosurg Rev 2011;34:393-407.

55. Holste K, Sahyouni R, Teton Z, et al. Spasm Freedom Following Microvascular Decompression for Hemifacial Spasm: Systematic Review and Meta-Analysis. World Neurosurg 2020;139:e383-90.

56. Bartindale $M$, Kircher $M$, Adams W, et al. Hearing Loss following Posterior Fossa Microvascular Decompression: A Systematic Review. Otolaryngol Head Neck Surg 2018;158:62-75. 


\section{Supplementary}

Table S1 Outcomes at different follow-up time points

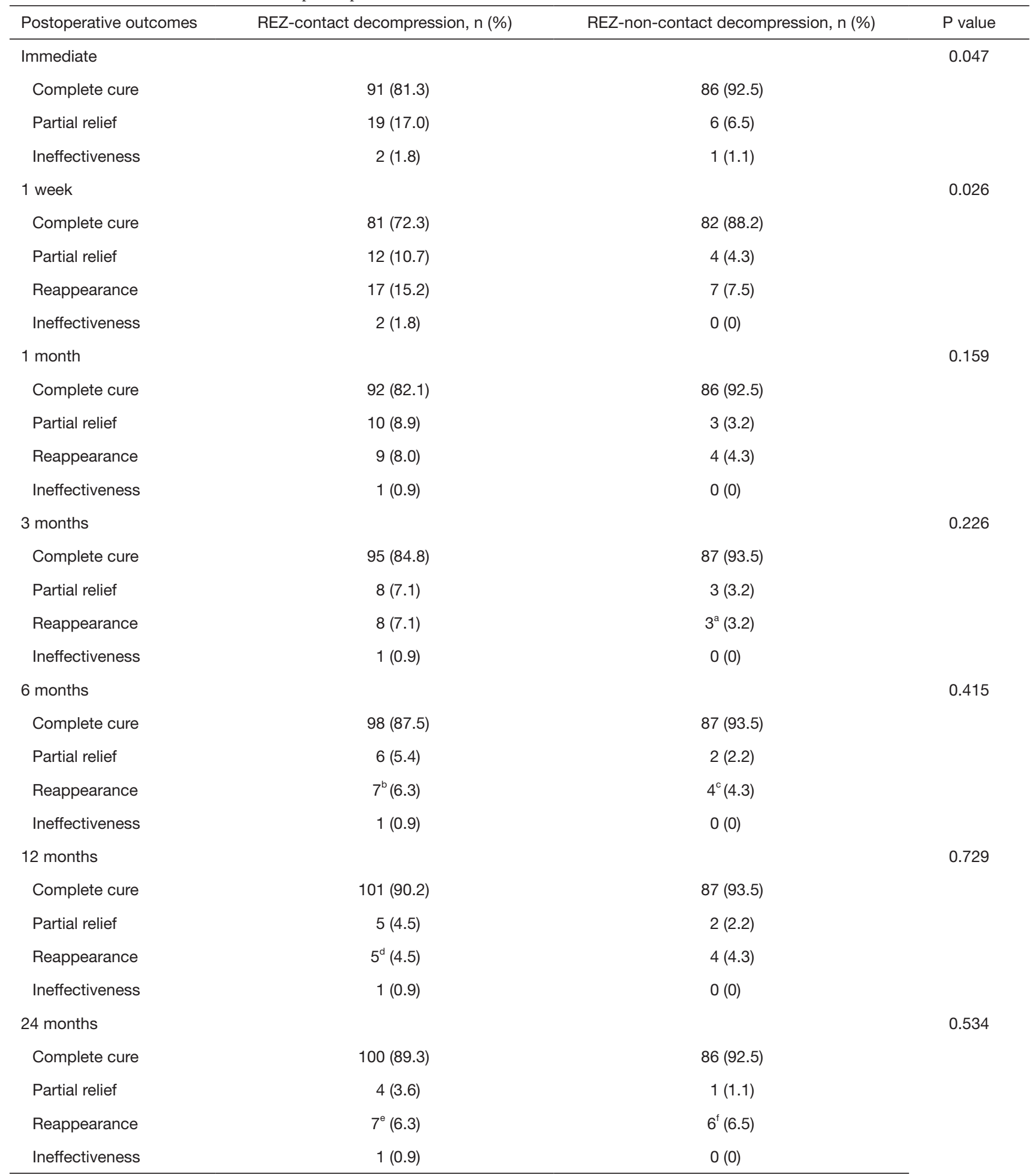

Delayed reappearance: ${ }^{a}$, blepharospasm re-emerged in 1 case at 3 months; ${ }^{b}, 1$ case relapsed at 5 months; ${ }^{c}, 2$ cases relapsed at $3-$ 6 months; ${ }^{d}, 3$ cases relapsed at $6-12$ months; ${ }^{e}, 2$ cases relapsed after 12 months; ${ }^{\dagger}, 2$ cases relapsed after 12 months. REZ, facial root entry/exit zone. 
Table S2 Complete cure at different follow-up time points

\begin{tabular}{lccc}
\hline \multirow{2}{*}{ Follow-up time points } & \multicolumn{3}{c}{ Complete cure, $\mathrm{n}(\%)$} \\
\cline { 2 - 3 } & $91(81.3)$ & \multicolumn{3}{c}{ REZ-non-contact decompression } \\
\hline Immediate & $81(72.3)$ & $86(92.5)$ & 0.020 \\
1 week & $92(82.1)$ & $82(88.2)$ & 0.005 \\
1 month & $95(84.8)$ & $86(92.5)$ & 0.029 \\
3 months & $98(87.5)$ & $87(93.5)$ & 0.049 \\
6 months & $101(90.2)$ & $87(93.5)$ & 0.146 \\
12 months & $100(89.3)$ & $87(93.5)$ & 0.384 \\
24 months & & $86(92.5)$ & 0.433 \\
\hline
\end{tabular}

REZ, facial root entry/exit zone.

Table S3 Partial relief at different follow-up time points

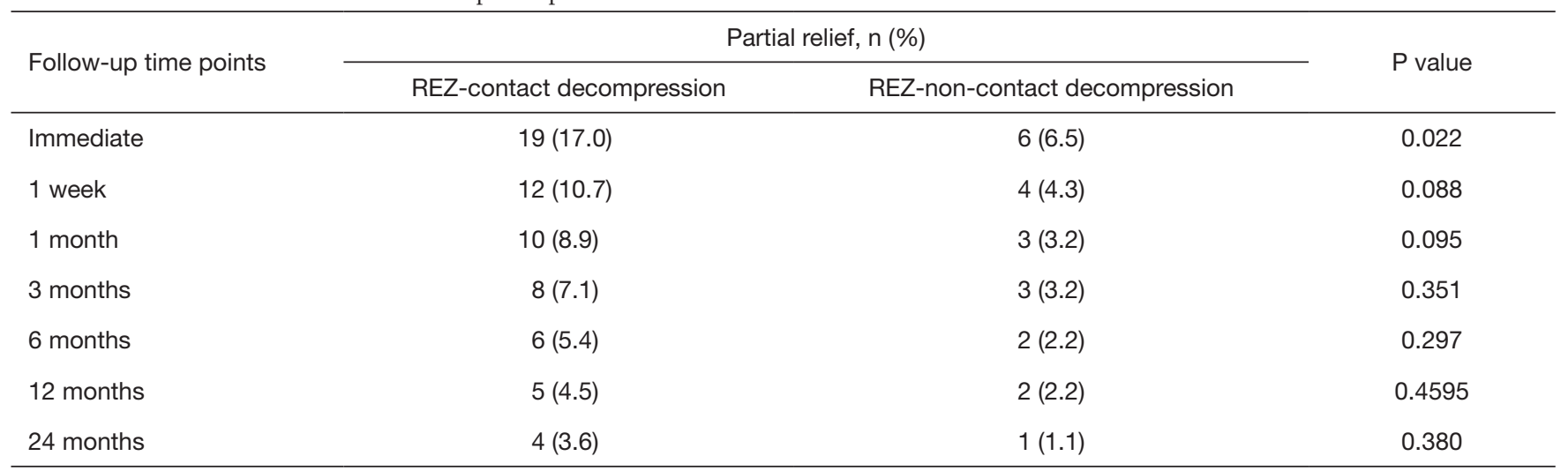

REZ, facial root entry/exit zone.

Table S4 Cumulative reappearance after the initial complete cure

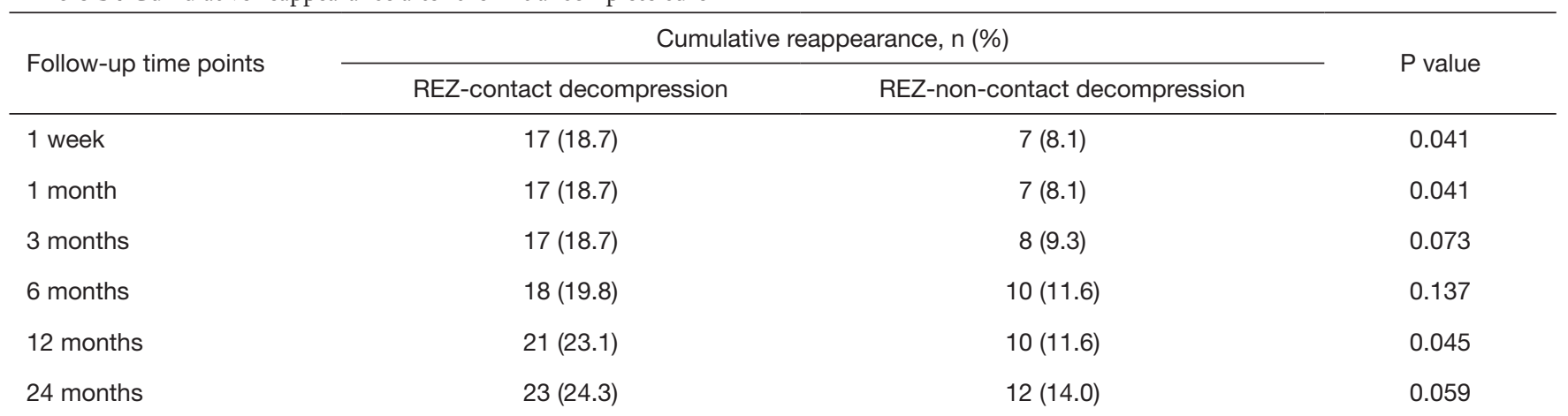

REZ, facial root entry/exit zone. 